\title{
EL SÍNDROME DEL «BURNOUT» EN PROFESORES DE EDUCACIÓN SECUNDARIA DE LIMA METROPOLITANA
}

\begin{abstract}
Ana Delgado Vásquez ${ }^{1}$
Se realizó el estudio del síndrome del «Burnout» en profesores de educación secundaria de Lima Metropolitana considerando las variables: sexo, tipo de colegio y tiempo de servicio. Participaron en la investigación 764 docentes de secundaria de las siete USEs de Lima Metropolitana, de centros educativos estatales y no estatales. Los resultados del análisis psicométrico del test demuestran que el instrumento permite obtener puntajes confiables, a través del método de consistencia interna. Asimismo, el análisis de factores permitió obtener la validez de constructo de la prueba, observándose que los Ítemes conforman un solo factor que explica el $55.54 \%$ de la varianza de las puntuaciones. Se elaboraron los baremos correspondientes. El análisis de los resultados destaca la comparación en los niveles de burnout y las diferencias en las fases del burnout según Golembiewski entre los docentes varones y mujeres, los profesores de colegios estatales y los no estatales, y considerando el tiempo de servicio.
\end{abstract}

PALABRAS CLAVE: Síndrome del Burnout, cansancio emocional, despersonalización, realización personal, niveles, fases de Golembiewski.

It was made a study of burnout syndrome in high school teachers of Lima, Perú, considering sex, school clasification and year at work. The participants were 764 high school teachers obtained at random considering all school districts (USE) of the city of Lima, Peru. They were teachers of public and private schools. The psychometric analysis shows that the item-test relation is up to norms $(>0.20)$, so reliability was obtained. Also the factor analysis of the items comes up whith one factor explaining the $55.54 \%$ of variance, so construct validity was obtained. Also the calification norms was calculated. The analysis of results points out the comparison considering the levels of burnout and the phases of burnout using. Golembiewski index, between male and female teachers, teachers from public and private schools, and teachers at work.

KEY WORDS: Burnout syndrome, emotional exhaustion, depersonalization, personal accomplishment, levels, Golembiewski phases.

${ }^{1}$ Miembros de la Investigación: Luis Miguel Escurra Mayaute, María Clotilde Atalaya Pisco, Carmen Alvarez Taco, Mario Bulnes Bedón, Manuel Rodomiro Campos Roldán, Juan Pequeña Constantino, Renato Santivañez Olulo. William Torres A. 


\section{INTRODUCCIÓN}

El síndrome del Burnout equivale a síndrome de desgaste profesional, síndrome o depresión por agotamiento. Es un deterioro o agotamiento producido por la demanda excesiva de recursos físicos y emocionales que lleva consigo cada profesión, se observa en profesiones que implican relaciones interpersonales intensas y continuas. El profesional se encuentra desbordado, agotada su capacidad de reacción. Asimismo, repercute intensamente, en el medio familiar y social, llegando en algunos casos a situaciones de alto riesgo.

El término Burnout (quemarse o quemazón) fue usado por primera vez por H.J. Freudenberger (psicólogo clínico familiar) en el año 1974, quien lo definía como fallar, desgastarse o sentirse exhausto debido a las demandas excesivas de energía, fuerza o recursos. Además señala que esto sucede cuando un miembro de una organización por las razones que fuesen y luego de muchos intentos se vuelve inoperante.

Si bien Freudenberger originó el concepto, fue la investigadora C. Maslach quien en 1982 definió dicha condición de una forma contundente. Actualmente se reconoce la definición establecida por Maslach como una de las más apropiadas, esta investigadora lo considera un síndrome de fatiga emocional, despersonalización y de un logro personal reducido, que puede ocurrir entre los individuos que trabajan directamente con pacientes. Asimismo, considera que es una respuesta a una tensión emocional de índole crónico, originada por el deseo de lidiar exitosamente con otros seres humanos que tienen problemas. Lo que es único de esta condición, es que la tensión surge de la interacción social entre la persona que recibe la ayuda y el que ayuda.

En la CIE 10 (Décima revisión de la Clasificación Europea de las Enfermedades) figura como síndrome de agotamiento dentro de un grupo de clasificación que tiene como título «problemas respecto a las dificultades para afrontar la vida» (Z 73.0).

Este síndrome aparece progresivamente como reflejo de un esfuerzo de adaptación inadecuado. Debe entenderse como una respuesta al estrés laboral que aparece cuando fallan las estrategias funcionales de afrontamiento que suele emplear el sujeto. No se trata de un estado, sino de un proceso crónico, con sintomatología intermitente o continua.

Con los sistemas de evaluación se han identificado tres dimensiones del síndrome:

a. Cansancio o agotamiento emocional: se define como cansancio y fatiga que puede manifestarse física, psíquicamente o como una combinación de ambos. La persona siente que ya no puede dar más de sí misma a los demás.

b. Despersonalización: se entiende como el desarrollo de sentimientos, actitudes y respuestas negativas, distantes y frías hacia otras personas, especialmente hacia los beneficiarios del propio trabajo. Se acompaña de un incremento en la irritabilidad y una pérdida de la motivación hacia si mismo. El profesional trata de distanciarse no sólo de los destinatarios de su trabajo sino también de los miembros del equipo con el que trabaja, mostrándose cínico, irónico e incluso utilizando a veces etiquetas despectivas para aludir a los usuarios y tratando de hacerles culpables de sus frustraciones y descenso del rendimiento laboral.

c. Baja realización personal: surge cuando se verifica que las demandas que se le hacen, exceden su capacidad para atenderlas de forma competente. Supone respuestas negativas hacia uno mismo y hacia su trabajo, evitación de las relaciones. personales y profesionales, bajo rendimiento laboral, incapacidad para soportar la presión y una baja autoestima. La falta de logro personal en el trabajo se caracteriza por una dolorosa desilusión y fracaso en dar sentido personal al trabajo. Se experimentan sentimientos de fracaso personal (falta de competencia, de esfuerzo o conocimientos), carencias de expectativas y horizontes en el trabajo, y una insatisfacción generalizada. Como consecuencia, la impuntualidad, la 
abundancia de interrupciones, la evitación del trabajo, el ausentismo y el abandono de la profesión, son síntomas habituales.

Gil y Peiró (1997) han afirmado que el síndrome del quemado puede estudiarse desde dos perspectivas: clínica y psicosocial. La perspectiva clínica asume el burnout como un estado (concepción estática) al que llega el sujeto como consecuencia del estrés laboral; y la psicosocial, lo define como un proceso con una serie de etapas que se generan por interacción de las características personales y del entorno laboral.

Como se señaló, desde una perspectiva clínica, Freudenberger (1974), empleó por primera vez el término burnout para describir un conjunto de síntomas físicos sufridos por personal que labora en servicios de ayuda como resultado de las condiciones de trabajo, y caracterizado por un estado de agotamiento que surge como resultado de trabajar de manera intensa, sin considerar las propias necesidades. Para este enfoque, el burnout aparece con mayor frecuencia, en los profesionales más comprometidos, en los que trabajan más ante la presión y demandas de su trabajo, relegando a un segundo plano sus intereses, resultando una relación inadecuada entre profesionales, excesivamente celosos en su trabajo y pacientes excesivamente necesitados, esto es, una respuesta o esfuerzo desmesurado por ayudar a los pacientes.

Desde esta perspectiva el síndrome de burnout estaría referido a la experiencia de agotamiento, decepción y pérdida de interés por la actividad laboral, que surge en los profesionales que trabajan en contacto directo con personas en la prestación de servicios y como consecuencia de ese contacto diario con su trabajo. Este estado es el resultado de «gastarse» por la prestación persistente de los servicios referidos y de un conjunto de expectativas inalcanzables (Freudenberguer, 1975).

Pines y Aronson en 1988 (Gil y Peiró, 1997) lo definen como un estado en el que se combinan la fatiga emocional, física y mental, sentimientos de impotencia e inutilidad y baja autoestima, acompañado de sentimientos de desamparo y desesperanza, desilusión y una actitud negativa hacia el trabajo y hacia la vida en general.

En la perspectiva psicosocial se encuentran los estudios de Maslach y Jackson (1981, 1985, 1986), quienes consideran el burnout como una respuesta emocional, situando los factores laborales y organizacionales como condicionantes y antecedentes. Estos autores se interesaron en investigar la forma en que el estrés relacionado con el rol laboral puede llevar a una persona a experimentar fatiga mental, a dar un trato «mecanizado» a los clientes y autopercibir disminuida su habilidad para lograr éxito en el trabajo. Definen el burnout como un síndrome tridimensional que se desarrolla en aquellos profesionales cuyo objeto de trabajo son personas (usuarios). Las tres dimensiones son: agotamiento emocional, despersonalización y falta de realización personal en el trabajo. Este síndrome es causado por estresores organizacionales, y se puede desarrollar en personas cuyo objeto de trabajo son también personas. Para ellos el burnout provoca en el trabajador una tendencia a tratar a sus «clientes» de una manera desprendida y deshumanizada.

Golembiewski, R.T., Munzenrider, R y Carter, D. (1983), Golembiewski, R.T., Munzenrider, R y Stevenson, J.G. (1986), Golembiewski, R.T. y Runtree, B. (1991), aseguran que el burnout afecta a todo tipo de profesiones y no sólo a las organizaciones de ayuda, gran parte de sus investigaciones se han llevado a cabo con muestras de directivos, vendedores, etc. Para estos investigadores el síntoma inicial del síndrome es el desarrollo de actitudes de despersonalización, un mecanismo disfuncional de afrontamiento del estrés. Posteriormente los profesionales experimentan baja realización personal en el trabajo y a más largo plazo agotamiento emocional. Consideran que existen ocho fases en el desarrollo del burnout, la combinación de la puntuación en las tres escalas determina la fase a la que es asignado el sujeto. Así, un sujeto que en las tres escalas puntúa por debajo de la media se considera que está en la primera fase del burnout, si puntúa superior a la media en 
despersonalización, pero inferior en falta de realización personal en el trabajo y agotamiento emocional se considera en la segunda fase y así sucesivamente hasta llegar a la fase ocho en la que el sujeto puntúa por encima de la media en las tres subescalas del MBI. Existen diferentes trabajos empíricos que han dado apoyo a la secuencia de fases del modelo en diferentes profesiones y en diferentes países. Para desarrollar el síndrome, los sujetos no tienen que pasar necesariamente por todas las fases establecidas en el modelo. Según el número de fases que atraviesa un sujeto se puede distinguir entre proceso agudo y proceso crónico. La instauración aguda del síndrome sería aquella en la que un sujeto pasa por ejemplo, por las fases I, V Y VIII, mientras que en la instauración crónica correspondería a una situación en la que el sujeto pasa por ejemplo, por las fases I, II, IV y VIII. Se identifica la fase IV como la fase crítica, p0.rque es en esta fase donde la persona comienza a experimentar los efectos del desgaste. Los propios autores señalan como defecto del modelo que no queda clara la forma en que un sujeto se recupera desde la fase VIII, si de forma aguda o crónica. Por otra parte, Golembiewski y col. piensan que las tres dimensiones no contribuyen por igual al síndrome del burnout. Así, mientras que los sentimientos de despersonalización son considerados como la variable que menos contribuye, la falta de realización personal en el trabajo es considerada como un contribuyente de peso más moderado, y los sentimientos de agotamiento emocional son considerados como la variable que más contribuye a que un sujeto desarrolle el síndrome.

Para Gil y Peiró (1997) el síndrome de burnout queda establecido como una respuesta al estrés laboral crónico integrado por actitudes y sentimientos negativos hacia las personas con las que se trabaja y hacia el propio rol profesional, así como por un sentimiento de encontrarse emocionalmente exhausto. Esta respuesta se da, frecuentemente, en las organizaciones de servicios, aunque no está restringida a ellas. Desde una perspectiva organizacional, Cherniss (1980) ha investigado cómo las organizaciones y sus ambientes socioculturales afectan la respuesta de una persona al trabajo. Considera que el agotamiento emocional, la despersonalización y la disminución de los logros personales son tres mecanismos para afrontar el trabajo estresante, frustrante o monótono.

Considerando la perspectiva sociohistórica Sarason en 1983 (Vandenberghe y Huberman, 1999) enfatiza el impacto de la sociedad en el desarrollo del burnout y no el efecto individual o de la organización. También señala que cuando las condiciones sociales no conducen a una preocupación por los otros, es difícil mantener un compromiso con trabajos relacionados al servicio social.

En lo que no existen dudas es en el hecho que el síndrome de burnout presenta alteraciones físicas, comportamentales y emocionales que tienen su origen en factores individuales, laborales y sociales. Los síntomas han sido agrupados en cuatro áreas sintomatológicas: síntomas psicosomáticos, síntomas conductuales, manifestaciones emocionales y síntomas defensivos (Maslach y Pines, 1977; Chermiss, 1980; y Maslach, 1982). Entre los síntomas psicosomáticos destacan: dolores de cabeza, fatiga crónica, úlceras o desórdenes gastrointestinales, dolores musculares en la espalda y cuello, hipertensión y en las mujeres pérdida del ciclo menstrual. En cuanto a síntomas conductuales se tiene el ausentismo laboral, conducta violenta, abuso de drogas, incapacidad de relajarse, etc. Entre las manifestaciones emocionales se pueden observar: el distanciamiento afectivo que el profesional manifiesta a las personas a las que atiende, la impaciencia, los deseos de abandonar el trabajo y la irritabilidad, dificultad para concentrarse debido a la ansiedad experimentada, produciéndose así un descenso del rendimiento laboral, surgen dudas acerca de la propia competencia profesional, generando el descenso de la autoestima. Finalmente, los autores señalan los síntomas defensivos que aluden a la negación emocional. Se trata de un mecanismo que utiliza el profesional para poder aceptar sus sentimientos, negando las emociones anteriormente descritas cuyas formas más habituales 
son: negación de las emociones, ironía, atención selectiva y el desplazamiento de sentimientos hacia otras situaciones o cosas. También pueden utilizar para defenderse la intelectualización o la atención parcial hacia lo que le resulta menos desagradable (Alvarez y Fernández, 1991).

El trabajo docente en el nivel secundario es una actividad vinculada a horarios fijos, sueldos establecidos y tareas rutinarias, con períodos sobrecargados de trabajo. Cabe indicar que buena parte del trabajo (preparación de clases, materiales, calificación de trabajos y exámenes, etc.), tiene que ser realizado en casa, fuera del horario de trabajo, lo cual no es reconocido ni en términos remunerativos, ni socialmente. Esta situación de trabajar con permanente dedicación y esfuerzo, teniendo escaso prestigio

social y con remuneraciones muy por debajo de lo que corresponde, es muy probable que esté generando alteraciones físicas, comportamentales y emocionales, por lo cual en el presente estudio se indaga sobre el síndrome del burnout en los docentes de educación secundaria de Lima Metropolitana, considerando el sexo, el tipo de colegio, y el tiempo de servicio.

\section{OBJETIVOS}

a. Determinar las normas del Inventario de Maslach en profesores de educación secundaria de Lima Metropolitana.

b. Conocer las características del síndrome de burnout en docentes de educación secundaria de Lima Metropolitana.

c. Precisar la interacción entre el síndrome de burnout y la actividad docente de los profesores de educación secundaria de Lima Metropolitana.

d. Analizar las características del burnout en función a las variables de género, tipo de colegio de procedencia y años de servicio de los docentes de educación secundaria de Lima Metropolitana.

e. Formular indicaciones con respecto al síndrome de burnout y la actividad de los profesores de secundaria de Lima Metropolitana.

\section{HIPÓTESIS}

H1: Existen diferencias en los ni veles de burnout si se considera el género en docentes de educación secundaria de Lima Metropolitana.

$\mathrm{H} 2$ : Existen diferencias en los niveles de burnout si se considera el tipo de colegio en el que laboran los docentes de educación secundaria de Lima Metropolitana.

H3: Existen diferencias en los niveles de burnout si se consideran los años de servicio de los profesores de educación secundaria de Lima Metropolitana.

\section{MÉTODO}

\section{Participantes}

El universo de investigación estuvo conformado por 30,456 docentes de educación secundaria en ejercicio en el año 2002 en los diferentes centros educativos de Lima Metropolitana. De acuerdo a los datos brindados por el Ministerio de Educación la población se encontraba distribuida de la siguiente manera: 
Tabla 1. Composición de la población por USE y tipo de colegio.

\begin{tabular}{cccl} 
USE & \multicolumn{2}{c}{ Tipo de Colegio } & Tot \\
& Estatal & No & al \\
01 & 1601 & 859 & 2460 \\
02 & 3372 & 1309 & 4681 \\
03 & 3229 & 1743 & 4972 \\
04 & 2938 & 2836 & 5774 \\
05 & 2247 & 1201 & 3448 \\
06 & 2021 & 836 & 2857 \\
07 & 1623 & 1008 & 2631 \\
Departamen & 1653 & 1980 & 3633 \\
Total & 18684 & 11772 & 3045 \\
& & &
\end{tabular}

El tamaño de la muestra se determinó utilizando el programa informático SOTAM, el cual determinó una muestra de 764 docentes distribuidos de la siguiente manera:

Tabla 2. Composición de la muestra por USE y tipo de colegio

\begin{tabular}{cccc} 
USE & \multicolumn{2}{c}{ Tipo de Colegio } & Total \\
& Estatal & No & \\
01 & 40 & 22 & 62 \\
02 & 85 & 33 & 117 \\
03 & 81 & 44 & 125 \\
04 & 74 & 71 & 145 \\
05 & 56 & 30 & 86 \\
06 & 51 & 21 & 72 \\
07 & 41 & 25 & 66 \\
Departamen & 41 & 50 & 91 \\
Total & 469 & 295 & 764 \\
Total & & &
\end{tabular}

Para la obtención de la muestra se llevó a cabo un procedimiento de muestreo probabilístico bi-etápico. En la primera etapa en cada una de las USEs se elegió de forma aleatoria los colegios a ser incluidos en el estudio y posteriormente en cada colegio se eligieron de forma aleatoria los participantes que fueron evaluados.

\section{Instrumento}

Se utilizó la adaptación española del Inventario de Maslach y Jackson o M.B.l. (1997) con el fin de evaluar las tres dimensiones del síndrome: Agotamiento emocional, Despersonalización, Realización Personal. El instrumento está constituido por 22 Ítemes que se valoran con una escala tipo Likert de 7 puntos.

La subescala de cansancio o agotamiento emocional (e E) comprende 9 Ítemes que describen los sentimientos de una persona emocionalmente exhausta por el trabajo. La subescala de despersonalización (DP) comprende 5 Ítemes que describen una respuesta impersonal y fría hacia los receptores de los servicios o cuidados del profesional. Tanto en ésta como en la escala anterior, los profesionales con puntuaciones 
Tabla 3. Análisis de la confiabilidad de la subescala Cansancio Emocional del Inventario «Burnout» de Maslach.

\begin{tabular}{|c|c|c|c|}
\hline Item & Media & $\mathbf{r}_{\text {itc }}$ & $\begin{array}{c}\text { Alfa } \\
\text { eliminando el } \\
\text { item }\end{array}$ \\
\hline 1 & 1.6348 & $.5382^{* * *}$ & .7559 \\
\hline 2 & 2.6348 & $.5400^{* * *}$ & .7637 \\
\hline 3 & 1.5877 & $.4279^{* * *}$ & 7543 \\
\hline 6 & 2.0497 & $.5680^{* * *}$ & .7763 \\
\hline 8 & 0.8455 & $.4624^{* * *}$ & .7533 \\
\hline 13 & 0.6322 & $.4137^{* * *}$ & .7679 \\
\hline 14 & 2.4136 & $.4749^{* * *}$ & .7800 \\
\hline 16 & 1.1283 & $.5312^{* * *}$ & .7569 \\
\hline 20 & 0.4961 & $.4282^{* * *}$ & .7717 \\
\hline
\end{tabular}

$* * * \mathrm{p}<.001 \mathrm{n}=764$

Alfa $=0.78$

En lo que concierne al análisis de Ítemes de la subescala despersonalización, en la tabla $\mathrm{N}^{\circ} 4$, se observó que todas las correlaciones Ítem-test corregidas también son superiores al criterio de 0.20 , lo cual corrobora que todos los Ítemes son consistentes entre sí.

El análisis de la confiabilidad de la segunda subescala, permite apreciar que se alcanza un coeficiente de Alfa de Cronbach de 0.71, lo que corrobora la confiabilidad de la subescala de despersonalización.

Tabla 4. Análisis de la confiabilidad de la subescala Despersonalización del Inventario «Burnout» de Maslach

\begin{tabular}{|c|c|c|c|}
\hline Item & Media & $\mathbf{r}_{\text {itc }}$ & $\begin{array}{c}\text { Alfa } \\
\text { eliminando el } \\
\text { item }\end{array}$ \\
\hline 5 & 0.4830 & $.3041 * * *$ & .7362 \\
\hline 10 & 0.9136 & $.2182 * * *$ & .7311 \\
\hline 11 & 0.9293 & $.3134 * * *$ & .7208 \\
\hline 15 & 1.8691 & $.4377 * * *$ & .7382 \\
\hline 22 & 0.9866 & $.4869 * * *$ & .7234 \\
\hline \multicolumn{4}{|c|}{$\mathrm{Alfa}=0.71$} \\
*** $\mathrm{p}<.001 \mathrm{n}=764$
\end{tabular}

En lo que respecta al análisis de Ítemes de la subescala realización personal (ver tabla N"5), se encontró que todas las correlaciones ítem-test corregidas calculadas son superiores al criterio de 0.20 , lo cual permite concluir que todos los Ítemes son consistentes entre sí.

El análisis de la confiabilidad de la subescala realización personal, permite apreciar que se obtiene un coeficiente Alfa de Cronbach de 0.76, lo cual permite indicar que la tercera subescala del instrumento es confiable. 


\section{Análisis de la Validez de Constructo}

Los resultados del análisis de la validez de constructo, efectuado a través del análisis factorial de las subescalas del Inventario «Burnout» de Maslach (ver Tabla $N^{\circ} 6$ ), permitió apreciar que se alcanza una medida de adecuación del Muestreo de Kaiser-Meyer-Olkin de $0.61 \mathrm{y}$ un test de esfericidad de Bartlett que es significativo, hallazgos que corroboran la pertinencia de la ejecución del análisis factorial. Este análisis se desarrolló a través del método de factorización de los componentes principales, notándose la existencia de un solo factor, el cual permite explicar el $55.54 \%$ de la varianza de las altas presentan grados elevados de vivencia del síndrome de burnout. De otro lado, la subescala de logro personal (PA) está constituida por 8 ítemes que describen sentimientos de competencia y realización exitosa en el trabajo hacia los demás. A diferencia con las otras dos escalas, las puntuaciones bajas son indicativas del síndrome (Maslach y Jackson, 1997).

En la versión española se reporta que en los estudios originales se llevaron a cabo la estimación de la confiabilidad por el método de consistencia interna mediante el Alfa de Cronbach en' una muestra de 1,316 casos, obteniéndose los siguientes índices:

Alfa $=0.90$ para cansancio o agotamiento emocional $(\mathrm{CE})$, Alfa $=0.79$ para despersonalización $(\mathrm{DP}), \mathrm{y}$ Alfa $=0.71$ para realización personal $(\mathrm{RP})$.

En otros dos análisis se empleó el procedimiento test re-test. En el primero se empleó una muestra de 53 graduados universitarios en servicios sociales con un intervalo de dos a cuatro semanas entre ambas aplicaciones, y los índices de confiabilidad fueron de 0,82 en CE, 0,60 en DP y 0,80 en RP. En el segundo estudio, sobre una muestra de 248 profesores $\mathrm{y}$ un intervalo de un año entre el momento del test y el del re-test, se hallaron los siguientes índices: 0,60 en CE, 0,54 en DP y 0,57 en RP. Todos estos índices son de tipos moderados pero significativos al nivel de confianza del $\mathrm{r} \%$.

En cuanto a la validez del instrumento tanto en los estudios factoriales, originales y españoles, se ha visto que los elementos que componen el MBI definen una estructura tridimensional que apunta a esas mismas dimensiones. Este tipo de validez factorial es apoyado por estudios de validez convergente, llevados a cabo por Maslach y Jackson (1986), quienes relacionaron las puntuaciones del MBl con:

- Las evaluaciones del comportamiento hechas por una persona que conoce bien al sujeto examinado (su pareja o un compañero en el puesto de trabajo).

- La presencia de algunas características laborales que normalmente provocan estrés.

- Las medidas en otras variables que, por hipótesis, están relacionadas con este estrés.

En los tres tipos de análisis se encontraron índices significativos al nivel de confianza del $5 \%$ y del $1 \%$ respectivamente.

\section{RESULTADOS}

\section{Análisis de la confiabilidad}

A continuación se presentan los resultados de los análisis de ítemes y los coeficientes de Con fiabilidad de las subescalas del Inventario «Burnout» de Maslach.

En el análisis de ítemes de la subescala cansancio emocional (ver tabla $\mathrm{N}^{\circ} 3$ ), se encontró que todas las correlaciones ítem-test corregidas son superiores al criterio propuesto por Kline (1986) de ser mayores a 0.20 , lo cual indica que todos los ítemes son consistentes entre sí.

El análisis de la confiabilidad de esta primera subescala, mostró un coeficiente Alfa de Cronbach de 0.78 , lo que permite corroborar la con fiabilidad de la subescala cansancio emocional.

puntuaciones. Este hallazgo permite concluir que los aspectos evaluados por las tres 
subescalas corresponden a un solo constructo que es el de «Burnout», por lo tanto la prueba presenta validez.

Tabla 6. Análisis de la validez de constructo del Inventario «Burnout» de Maslach a través del análisis factorial.

\begin{tabular}{|l|l|l|l|}
\hline Sub- escalas & M & D.E & Factor \\
\hline Cansancio emocional & 1.49 & 1.02 & 0.78 \\
Despersonalización & 0.82 & 0.91 & 0.80 \\
Realización personal & 4.83 & 0.91 & 0.64 \\
Varianza explicada & & & $55.54 \%$ \\
\hline
\end{tabular}

Medida de adecuación del muestreo de Kaiser-Meyer-Olkin;

0.61 Test de esfericidad de Bartlett; $250.84 * * *$

$* * * \mathrm{p}<.001 \mathrm{n}=764$

\section{Baremos de la prueba}

En el anexo I se incluye la tabla de los rangos percentiles de cada una de las tres subescalas del Inventario «Burnout» de Maslach para los docentes varones y mujeres de colegios estatales y no estatales de Lima Metropolitana.

\section{Análisis de la Contrastación de hipótesis}

Antes de efectuar el análisis de la contrastación de las hipótesis planteadas, se procedió a verificar la existencia de distribuciones normales en los puntajes de las subescalas que componen el inventario, con la finalidad de seleccionar el análisis estadístico más adecuado a la calidad del dato obtenido. Los resultados observados en la tabla $\mathrm{N}^{\circ}$, indicaron que todos valores del estadístico $\mathrm{Z}$ de Kolmogorov Smirnov son significativos $(p<.001)$ concluyéndose por tanto que los datos no se aproximan adecuadamente a la distribución normal, por lo que se aplicaron estadísticos no-paramétricos para realizar los contrastes.

Tabla 7. Análisis de la bondad de ajuste a la curva normal de las subescalas del Inventario «Burnout» de Maslach.

\begin{tabular}{|l|l|l|l|}
\hline Sub- escalas & $\mathrm{M}$ & $\mathrm{D} . \mathrm{E}$ & $\mathrm{KS}-\mathrm{Z}$ \\
\hline Cansancio emocional & 1.49 & 1.02 & $2.886 * * *$ \\
Despersonalizacion & 0.82 & 0.91 & $5.467 * * *$ \\
Realización personal & 4.83 & 0.91 & $3.373 * * *$ \\
\hline \multicolumn{4}{l}{$* * * \mathrm{p}<.001$} \\
$\quad \mathrm{~N}=764$
\end{tabular}

\section{Comparación por sexo}

En la comparación por sexo (Ver tabla $\mathrm{N}^{\circ} 8$ ), se observó que no existen diferencias estadísticas significativas entre los profesores y las profesoras, en ninguna de las tres subescalas del MBI. 
Tabla 8. Prueba U de Mann- Whitney del Inventario «Burnout» de Maslach considerando el sexo.

\begin{tabular}{|c|c|c|c|c|c|}
\hline subescala & $\begin{array}{l}\text { Varones } \\
\mathrm{N}=297 \\
\text { Media de } \\
\text { rangos }\end{array}$ & $\begin{array}{l}\text { Mujeres } \\
\mathrm{N}=467 \\
\text { Media de } \\
\text { rangos }\end{array}$ & $\mathrm{U}$ & $\mathrm{W}$ & $\mathrm{Z}$ \\
\hline Cansancio emocional & 394.91 & 374.61 & 65663.0 & 174941.0 & 1.2 \\
\hline Despersonalización & 401.27 & 370.56 & 63775.0 & 173053.0 & 1.9 \\
\hline Realización personal & 397.30 & 373.08 & 64952.0 & 174230.0 & 1.4 \\
\hline
\end{tabular}

$* \mathrm{p}<.05$

En la comparación por tipo de colegio (ver tabla $\mathrm{N}^{\circ}$ 9), se observo que existen diferencias significativas entre los docentes procedentes de colegios estatales y no estatles en la subescala cansancio emocional $(Z=-3.88 ; \mathrm{p}<.001)$, notándose que los docentes de centro educativos no estatales obtuvieron promedios de rangos mas altos que sus colegas de centros estatales. En la sub escala de despersonalización no se encontró diferencias significativas entre los docentes de ambos tipos de colegio. En cuanto a la subescala realización personal $(\mathrm{Z}=3.01 ; \mathrm{P}<.01)$, se pudo observar que existen diferencias significativas entre los docentes de colegios estatales y no estatales, siendo los docentes de colegios educativos no estatales, quienes obtuvieron promedio de rangos mas altos que los docentes de colegios no estatales.

Tabla 9. Prueba U de Mann-Whitney del Inventario «Burnout» de Maslach considerando tipo de colegio.

\begin{tabular}{|l|l|l|l|l|l|}
\hline subescala & $\begin{array}{l}\text { Estatal } \\
\mathrm{N}=468 \\
\text { Media de }\end{array}$ & $\begin{array}{l}\text { NoEstatal } \\
\mathrm{N}=296 \\
\text { Media de } \\
\text { rangos }\end{array}$ & $\mathrm{U}$ & $\mathrm{W}$ & $\mathrm{Z}$ \\
\hline Cansancio emocional & 357.286 & 421.46 & 57733.0 & 167479 & $-3.88 * * *$ \\
Despersonalización & 370.51 & 401.46 & 63651.0 & 173397 & -1.92 \\
Realización personal & 401.56 & 352.37 & 60345.0 & 104301 & $3.01 * *$ \\
\hline
\end{tabular}

$* * * \mathrm{p}<.001, * * \mathrm{p}<.01$

\section{Comparación por tiempo de servicio}

Con respecto a la comparación por tiempo de servicio, en la tabla 10, se observó que no existen diferencias estadísticamente significativas en ninguna de las subescalas del inventario considerando el tiempo de servicios de los docentes.

\section{Comparaciones complementarias por sexo y tipo de colegio}

Con respecto a la comparación por sexo y tipo de colegio, en la tabla $\mathrm{N}^{\circ} 11$, se observaron diferencias estadísticamente significativas entre los profesores y las profesoras de los colegios estatales y no estatales en las tres subescalas. Así en cuanto a la subescala can- 
sancio emocional se obtuvo un valor de Chi Cuadrada $\left(c^{2}=23.84 ; \mathrm{p}<.001\right)$, siendo las profesoras de colegios no estatales quienes obtuvieron una media de rangos más elevada. En despersonalización el valor de la Chi Cuadrada fue 13.02 ( $\mathrm{p}<.01$ ) nuevamente las docentes de colegios no estatales fueron las que obtuvieron un promedio de rangos superior. En cuanto a realización personal se observó un valor significativo en la Chi cuadrada $\left(\mathrm{c}^{2}=12.75 ; \mathrm{P}<.01\right)$ siendo los profesores de colegios estatales los que obtuvieron una media de rangos mayor.

Tabla 11. Prueba Kruskal-Wallis del Inventario «Burnout» de Maslach considerando el sexo y el tipo de colegio.

\begin{tabular}{|c|c|c|c|c|c|}
\hline \multirow[t]{2}{*}{ Subescalas } & $\begin{array}{c}\text { Varón } \\
\text { estatal } \\
\mathrm{N}= \\
176\end{array}$ & $\begin{array}{c}\text { Mujer } \\
\text { estatal } \\
\mathrm{N}= \\
292\end{array}$ & $\begin{array}{c}\text { Varón } \\
\text { no } \\
\text { estatal } \\
\mathrm{N}= \\
121 \\
\end{array}$ & $\begin{array}{c}\text { Mujer } \\
\text { no } \\
\text { estatal } \\
\mathrm{N}= \\
175 \\
\end{array}$ & \multirow[t]{2}{*}{$\mathbf{X}^{2}$} \\
\hline & $\begin{array}{l}\text { Media } \\
\text { de } \\
\text { rangos }\end{array}$ & $\begin{array}{l}\text { Media } \\
\text { de } \\
\text { rangos }\end{array}$ & $\begin{array}{l}\text { Media } \\
\text { de } \\
\text { rangos }\end{array}$ & $\begin{array}{l}\text { Media } \\
\text { de } \\
\text { rangos }\end{array}$ & \\
\hline Cansancio emocional & 391.86 & 337.37 & 399.35 & 436.74 & $23.84 * * *$ \\
\hline Despersonalización & 409.92 & 347.35 & 390.14 & 409.29 & $13.02 * *$ \\
\hline Realización personal & 408.59 & 397.32 & 380.90 & 332.64 & $12.75 * *$ \\
\hline
\end{tabular}

Tabla 12. Prueba U de Mann- Whitney del Inventario «Burnout» de Maslach considerando el sexo y tipo de colegio estatal.

\begin{tabular}{|c|c|c|c|c|c|}
\hline \multirow[t]{2}{*}{ Subescala } & $\begin{array}{c}\text { Varones } \\
\text { Estatal } \\
\mathrm{N}=468\end{array}$ & $\begin{array}{c}\text { Mujeres } \\
\text { Estatal } \\
\mathrm{N}=296\end{array}$ & \multirow[t]{2}{*}{$\mathbf{U}$} & \multirow[t]{2}{*}{$\mathbf{W}$} & \multirow[t]{2}{*}{$\mathbf{Z}$} \\
\hline & $\begin{array}{c}\text { Media de } \\
\text { rangos }\end{array}$ & $\begin{array}{c}\text { Media de } \\
\text { rangos }\end{array}$ & & & \\
\hline Cansancio emocional & 255.24 & 222.00 & 22045.0 & 64823 & $2.58 * *$ \\
\hline Despersonalización & 258.31 & 220.15 & 21505.0 & 64283 & $3.01 * *$ \\
\hline Realización personal & 238.53 & 232.07 & 24986.0 & 67764 & 0.50 \\
\hline
\end{tabular}

$$
* * p<.01
$$

Con respecto a la comparación por sexo considerando sólo a los docentes de colegios estatales, en la tabla 12, se observó que existen diferencias estadísticamente significativas entre los varones y las mujeres en las subescalas cansancio emocional $(\mathrm{Z}=2.57$; $\mathrm{P}<.01)$, $\mathrm{Y}$ despersonalización $(\mathrm{Z}=3.00 ; \mathrm{P}<.01)$. Observándose que en ambos casos son los varones los que obtuvieron promedios de rangos más elevados. En la subescala realización personal no se encontró diferencias significativas entre los docentes varones y mujeres de centros educativos estatales.

En la comparación de los indicadores del burnout por sexo entre los profesores de colegios no estatales, en la tabla $\mathrm{N}^{\circ} 13$, se observó que no existen diferencias estadísticamente significativas entre los varones y mujeres en ninguna de las tres subescalas. 
Tabla 13. Prueba U de Mann- Whitney del Inventario «Burnout» de Maslach considerando el sexo y tipo de colegio no estatal.

\begin{tabular}{|l|c|c|c|c|c|}
\hline \multicolumn{1}{|c|}{ Subescala } & $\begin{array}{c}\text { Varones } \\
\text { No } \\
\text { Estatal } \\
\mathrm{N}=176\end{array}$ & $\begin{array}{c}\text { Mujeres } \\
\text { No Estatal } \\
\mathrm{N}=292\end{array}$ & $\mathbf{U}$ & $\mathbf{W}$ & $\mathbf{Z}$ \\
& $\begin{array}{c}\text { Media de } \\
\text { rangos }\end{array}$ & $\begin{array}{c}\text { Media de } \\
\text { rangos }\end{array}$ & & & \\
\cline { 2 - 6 } & 139.85 & 154.48 & 9540.5 & 16921.5 & -1.45 \\
Cansancio emocional & 144.37 & 151.35 & 10088.0 & 17469. & -0.70 \\
Despersonalización & 159.82 & 140.67 & 9217.5 & 24617.0 & 1.90 \\
Realización personal & \multicolumn{7}{|c|}{$*$ p $<.05$}
\end{tabular}

Al compararse sólo a los docentes varones de colegios estatales y no estatales, en la tabla 14, se observó que no existen diferencias significativas en ninguna de las tres subescalas del inventario

Tabla 14. Prueba U de Mann-Whitney del Inventario «Burnout» de Maslach por tipo de colegio en docentes varones.

\begin{tabular}{|l|c|c|c|c|c|}
\hline \multirow{7}{*}{ Subescala } & $\begin{array}{c}\text { Varones } \\
\text { Estatal } \\
\text { N=176 }\end{array}$ & $\begin{array}{c}\text { Varones } \\
\text { No Estatal } \\
\text { N=121 }\end{array}$ & $\mathbf{U}$ & $\mathbf{W}$ & $\mathbf{Z}$ \\
\cline { 2 - 6 } & $\begin{array}{c}\text { Media de } \\
\text { rangos }\end{array}$ & $\begin{array}{c}\text { Media de } \\
\text { rangos }\end{array}$ & & & \\
\hline Cansancio emocional & 147.84 & 150.69 & 10443.0 & 26019.0 & -0.28 \\
Despersonalización & 151.74 & 145.01 & 10165.0 & 17546.0 & 0.67 \\
Realización personal & 193.51 & 158.39 & 12318.0 & 27718.5 & 1.10 \\
\hline
\end{tabular}

En la comparación por tipo de colegio en docentes mujeres, tabla $\mathrm{N}^{\circ} 15$, se observaron diferencias estadísticamente significativas en las subescalas cansancio emocional $(Z=-$ 4.71; $\mathrm{p}<.001)$, despersonalización $(\mathrm{Z}=-3.00 ; \mathrm{p}<.01)$, y realización personal $(\mathrm{Z}=3.03 ; \mathrm{p}<$ .01). Observándose que en las subescalas de cansancio emocional y despersonalización, las profesoras de colegios no estatales obtuvieron promedios de rangos más elevados, en cambio, son las docentes de colegios estatales quienes obtuvieron un promedio de rangos más elevado en realización personal.

Al comparar en los indicadores de burnout a los profesores de colegios estatales con las profesoras de colegios no estatales, tabla 16 , sólo se observaron diferencias significativas en la subescala realización personal $(\mathrm{Z}=3.24$; $\mathrm{P}<.01)$, siendo los varones de colegios estatales quienes obtuvieron una media de rangos más elevada. 
Tabla 15. Prueba U de Mann-Whitney del Inventario «Burnout» de Maslach en docentes mujeres considerando el tipo de colegio

\begin{tabular}{|c|c|c|c|c|c|}
\hline Subescala & $\begin{array}{c}\text { Mujeres } \\
\text { Estatal } \\
\mathrm{N}=292\end{array}$ & $\begin{array}{c}\text { Mujeres } \\
\text { No Estatal } \\
\mathrm{N}=175\end{array}$ & $\mathbf{U}$ & $\mathbf{W}$ & $\mathbf{Z}$ \\
& $\begin{array}{c}\text { Media de } \\
\text { rangos }\end{array}$ & $\begin{array}{c}\text { Media de } \\
\text { rangos }\end{array}$ & & & \\
\hline Cansancio & 211.24 & 271.98 & 10443.0 & 26019.0 & -0.28 \\
pmocional & 219.73 & 257.81 & 10165.0 & 17546.0 & 0.67 \\
Pespersonalización & 248.64 & 209.58 & 12318.0 & 27718.5 & 1.10 \\
$\begin{array}{c}\text { Realización } \\
\text { personal }\end{array}$ & & & & \\
\hline
\end{tabular}

Tabla 16. Prueba U de Mann- Whitney del Inventario «Burnout» de Maslach por tipo de colegio y sexo

\begin{tabular}{|l|c|c|c|c|c|}
\hline \multirow{1}{*}{ Subescala } & $\begin{array}{c}\text { Varones } \\
\text { Estatal } \\
\mathrm{N}=292\end{array}$ & $\begin{array}{c}\text { Mujeres } \\
\text { No Estatal } \\
\mathrm{N}=175\end{array}$ & $\mathbf{U}$ & \multirow{2}{*}{$\mathbf{W}$} & $\mathbf{Z}$ \\
\cline { 2 - 4 } & $\begin{array}{c}\text { Media de } \\
\text { rangos }\end{array}$ & $\begin{array}{c}\text { Media de } \\
\text { rangos }\end{array}$ & & & \\
\hline Cansancio emocional & 165.78 & 186.28 & 13601.5 & 29177.5 & -1.89 \\
Despersonalización & 175.87 & 176.13 & 15376.5 & 30952.5 & -0.25 \\
Realización personal & 193.51 & 158.39 & 12318.5 & 27718.5 & $3.25^{* *}$ \\
\hline
\end{tabular}

Cuando se comparó en los indicadores de burnout a las profesoras de centros educativos estatales con los profesores de centros educativos no estatales (ver tabla 17), se observaron diferencias estadísticamente significativas sólo en la subescala cansancio emocional $(\mathrm{Z}=-$ $2.61 ; \mathrm{p}<.01)$, siendo los varones de colegios no estatales quienes obtuvieron un promedio de rangos más elevado. En las subescalas despersonalización y realización personal no se encontraron diferencias significativas.

Tabla 17. Prueba U de Mann- Whitney del Inventario «Burnout» de Maslach por tipo de colegio y sexo.

\begin{tabular}{|l|c|c|c|c|c|}
\hline \multirow{7}{*}{ Subescala } & $\begin{array}{c}\text { Mujeres } \\
\text { Estatal } \\
\mathrm{N}=292\end{array}$ & $\begin{array}{c}\text { Varones } \\
\text { No Estatal } \\
\text { N =121 }\end{array}$ & $\mathbf{U}$ & $\mathbf{W}$ & $\mathbf{Z}$ \\
\cline { 2 - 4 } & $\begin{array}{c}\text { Media de } \\
\text { rangos }\end{array}$ & $\begin{array}{c}\text { Media de } \\
\text { rangos }\end{array}$ & & & \\
\hline Cansancio emocional & 165.78 & 186.28 & 13601.5 & 29177.5 & -1.89 \\
Despersonalización & 175.87 & 176.13 & 176.13 & 30952.5 & -0.25 \\
Realización personal & 193.51 & 158.39 & 158.39 & 27718.5 & $3.25^{* *}$ \\
\hline
\end{tabular}




\section{Fases del burnout según Golembiewski}

Los resultados indicaron que los 764 participantes, se distribuyen en las ocho fases del burnout que plantea Golembiewski (Tabla 18) de la siguiente manera: La mayor frecuencia en la distribución se encuentra en la fase 1, 193 docentes que representan el $25.3 \%$. Asimismo, se encontró que la segunda frecuencia más alta corresponde a la fase VIII (última fase), con 127 docentes (16.6\%). La fase V obtuvo la tercera frecuencia más alta, con 109 profesores que equivale al 14.3\%. Las fases II, III y VI, obtuvieron porcentajes sobre el $10 \%$ y las fases IV y VII obtuvieron porcentajes menores. Adicionalmente se realizó el análisis de las diferencias en la probabilidad de presentación de las fases del burnout y se encontró que existen diferencias significativas $\left(\mathrm{c}^{2}=164.82\right.$, g. $\left.1 .=7, \mathrm{P}<.001\right)$ notándose que predomina la presencia de las Fases 1 y VIII sobre las otras.

Tabla18. Distribución de los docentes en las fases del burnout según Golembiewski

\begin{tabular}{|c|c|c|c|}
\hline Fases & Frecuencia & Porcentaje (\%) & $\mathrm{X}^{2}$ \\
\hline I & 193 & 25.3 & \\
II & 79 & 10.3 & \\
III & 79 & 10.3 & \\
IV & 54 & 7.1 & \\
V & 109 & 14.3 & \\
VI & 77 & 10.1 & \\
VII & 46 & 6.0 & \\
VIII & 127 & 16.6 & $164.82 * *$ \\
\hline Total & 764 & 100.0 & \\
\hline
\end{tabular}

En la tabla 19 se presenta la distribución de los participantes en los tres niveles de burnout. Se puede observar que el $45.9 \%$ de estos profesores obtuvieron puntuaciones que los ubican en el nivel bajo. El $32.7 \%$ se encontró en el nivel alto y el $21.3 \%$ se ubicó en el nivel medio. También se llevó a cabo el análisis de las diferencias referida a la probabilidad de presentación de los niveles de burnout y se encontró que existen diferencias significativas $\left(\mathrm{e}^{2}=69.52\right.$, g.l. $\left.=2, \mathrm{P}<.001\right)$, notándose que predominan los niveles bajo y alto con respecto al nivel medio

Tabla 19. Clasificación de los docentes que presentaron el síndrome de burnout por niveles.

\begin{tabular}{|c|c|c|c|}
\hline nivel & Frecuencia & Porcentaje $(\%)$ & $\mathrm{X}^{2}$ \\
\hline Bajo & 351 & 45.9 & \\
Medio & 163 & 21.3 & \\
Alto & 250 & 32.7 & \\
\hline Total & 764 & 100.0 & $69.52 * * *$ \\
\hline \multicolumn{4}{|c|}{$* * * \mathrm{p}<001(\mathrm{gl}=2)$}
\end{tabular}

\section{Comparación de las fases y niveles del burnout por sexo}

En la comparación considerando el sexo de los docentes y las ocho fases del burnout

(Ver tabla 20), se observó que no existen diferencias estadísticas significativas entre los 
docentes varones y mujeres en las ocho fases descritas por Golembiewski.

Tabla 20. Prueba de Kruskal-Wallis de las ocho fases del burnout de Golembiewski considerando el sexo.

\begin{tabular}{|l|l|l|l|l|l|l|l|l|l|}
\hline Sexo & Fase & Fase & $\begin{array}{l}\text { Fase } \\
\text { I }\end{array}$ & $\begin{array}{l}\text { Fase } \\
\text { II }\end{array}$ & $\begin{array}{l}\text { Fase } \\
\text { III }\end{array}$ & $\begin{array}{l}\text { Fase } \\
\text { IV }\end{array}$ & Fase & Fase & $\mathrm{X}^{2}$ \\
& 76 & 34 & 24 & 15 & 50 & 29 & 12 & 57 & \\
\hline Varones & 117 & 45 & 55 & 39 & 59 & 48 & 34 & 70 & \\
Mujeres & 193 & 79 & 79 & 54 & 109 & 77 & 46 & 127 & 13.18 \\
Total & $* \mathrm{p}<.0 .5(\mathrm{gl}=7)$ \\
\hline
\end{tabular}

\section{Comparación de las fases del burnout por tipo de colegio}

En la comparación considerando el tipo de colegio donde laboran los docentes y las fases del burnout (ver tabla 21), se observó que existen diferencias significativas $\left(\mathrm{e}^{2}=36.98\right.$, g.1. $\left.=.7, \mathrm{p}<.001\right)$, notándose que predominan los profesores de colegios estatales en las fases del 1 al VI, mientras que los profesores de colegios no estatales predominan en la fase VIII del burnout según Golembiewski.

Tabla 21. Prueba de Kruskal- Wallis de las ocho fases del burnout de Golembiewski considerando el tipo de colegio

\begin{tabular}{|l|l|l|l|l|l|l|l|l|c|}
\hline $\begin{array}{l}\text { Tipo de } \\
\text { colegio }\end{array}$ & Fase & Fase & Fase & Fase & Fase & Fase & Fase & Fase & \multirow{2}{*}{$\mathrm{X}^{2}$} \\
\hline Estatal & 126 & 49 & 55 & 40 & 72 & 52 & 24 & 50 & \\
No estatal & 67 & 30 & 24 & 14 & 37 & 25 & 22 & 77 & \\
Total & 193 & 79 & 79 & 54 & 109 & 77 & 46 & 127 & $36.98 * * *$ \\
\hline \multicolumn{8}{|c|}{$* * 0.01(\mathrm{gl}=7)$}
\end{tabular}

\section{Comparación de las fases del burnout por tipo de colegio}

En la comparación considerando el tiempo de servicio del docente y las fases del burnout(ver tabla 22), se observó que no existen diferencias estadísticas significativas. 
Tabla 22. Prueba de Kruskal- Wallis de las ocho fases del burnout de Golembiewski considerando el tiempo de servicio.

\begin{tabular}{|c|c|c|c|c|c|c|c|c|c|}
\hline $\begin{array}{l}\text { Tiempo } \\
\text { de servicio }\end{array}$ & $\begin{array}{l}\text { Fase } \\
\text { I }\end{array}$ & $\begin{array}{l}\text { Fase } \\
\text { II }\end{array}$ & $\begin{array}{l}\text { Fase } \\
\text { III }\end{array}$ & $\begin{array}{l}\text { Fase } \\
\text { IV }\end{array}$ & $\begin{array}{l}\text { Fase } \\
\mathrm{V}\end{array}$ & $\begin{array}{l}\text { Fase } \\
\text { VI }\end{array}$ & $\begin{array}{l}\text { Fase } \\
\text { VII }\end{array}$ & $\begin{array}{l}\text { Fase } \\
\text { VIII }\end{array}$ & $\mathrm{X}^{2}$ \\
\hline 0.5 años & 84 & 32 & 34 & 23 & 51 & 33 & 25 & 63 & \\
\hline 6-10 años & 38 & 19 & 16 & 6 & 27 & 17 & 6 & 27 & \\
\hline 11-15 años & 35 & 17 & 11 & 14 & 13 & 14 & 7 & 24 & \\
\hline 16-20 años & 20 & 5 & 9 & 9 & 12 & 8 & 5 & 9 & \\
\hline 21 a más años & 16 & 6 & 9 & 2 & 6 & 5 & 3 & 4 & \\
\hline Total & 193 & 79 & 79 & 54 & 109 & 77 & 46 & 127 & 24.80 \\
\hline
\end{tabular}

\section{Contraste de Hipótesis}

El análisis de la primera hipótesis (H 1) en la que se compara a los profesores y profesoras considerando los tres nivelesde burnout, (Tabla 23), se encontró que no existen diferencias significativas entre varones y mujeres de los tres niveles, por lo cual esta hipótesis no es aceptada.

Tabla 23. Prueba de Kruskal- Wallis de los tres niveles del burnout considerando el sexo.

\begin{tabular}{|c|c|c|c|c|c|}
\hline Sexo & Nivel bajo & $\begin{array}{c}\text { Nivel } \\
\text { medio }\end{array}$ & $\begin{array}{c}\text { Nivel } \\
\text { alto }\end{array}$ & Total & $\mathrm{X}^{2}$ \\
\hline Varones & 134 & 65 & 98 & 297 & \\
Mujeres & 217 & 98 & 152 & 467 & \\
\hline Total & 351 & 163 & 250 & 764 & 0.15 \\
\hline \multicolumn{7}{|c}{$* \mathrm{p}<.05(\mathrm{gl}=2)$}
\end{tabular}

Analizando los resultados de la hipótesis 2, la cual considera el tipo de colegio donde laboran los docentes y las fases del burnout (ver tabla 24), se observó que existen diferencias significativas $\left(\mathrm{c}^{2}=18.93\right.$, g.l. $\left.=7, \mathrm{P}<.001\right)$. Se nota que en los profesores de colegios estatales predomina el nivel bajo de burnout mientras que en los profesores de colegios no estatales predominan los niveles bajo y alto, por lo que se concluye que esta hipótesis (H2) es aceptada.

Tabla 24. Prueba de Kruskal- Wallis de los tres niveles del burnout considerando el tipo de colegio

\begin{tabular}{|c|c|c|c|c|c|}
\hline $\begin{array}{l}\text { Tipo de } \\
\text { colegio }\end{array}$ & Nivel bajo & $\begin{array}{l}\text { Nivel } \\
\text { medio }\end{array}$ & $\begin{array}{c}\text { Nivel } \\
\text { alto }\end{array}$ & Total & $\mathrm{X}^{2}$ \\
\hline Estatal & 230 & 112 & 126 & 468 & \\
\hline No estatal & 121 & 51 & 124 & 296 & \\
\hline Total & 351 & 163 & 250 & 764 & $18.93 * * *$ \\
\hline
\end{tabular}


Al comparar la hipótesis referida el tiempo de servicio y los niveles del burnout de los docentes (Ver tabla 25), se observó que no existen diferencias significativas $\left(\mathrm{e}^{2}=7.50\right)$ por lo que se concluye que la tercera hipótesis (H3) no es aceptada.

Tabla 25. Prueba de Kruskal- Wallis de los tres niveles del burnout considerando el tiempo de servicio.

\begin{tabular}{|c|c|c|c|c|c|}
\hline $\begin{array}{l}\text { Tiempo de } \\
\text { servicio }\end{array}$ & Nivel bajo & $\begin{array}{c}\text { Nivel } \\
\text { medio }\end{array}$ & $\begin{array}{c}\text { Nivel } \\
\text { alto }\end{array}$ & Total & $\mathrm{X}^{2}$ \\
\hline 0-5 años & 150 & 74 & 121 & 345 & \\
6-10 años & 73 & 33 & 50 & 156 & \\
11-15 años & 63 & 27 & 45 & 135 & \\
16-20 años & 34 & 21 & 22 & 77 & \\
21 a más & 31 & 8 & 12 & 51 & 7.50 \\
\hline Total & 351 & 163 & 250 & 764 & \\
\hline
\end{tabular}

\section{DISCUSIÓN}

Los resultados de la adaptación del Inventario «Burnout» de Maslach muestran que la prueba es válida y confiable para los docentes de secundaria de centros educativos estatales y no estatales de Lima Metropolitana. Estos resultados son consistentes con lo hallado por Maslach y Jackson (1997), en diferentes profesionales españoles entre ellos profesores, así como por lo reportado por Fernández (2001) en docentes de educación primaria en Lima.

Al comparar a los docentes, se observó que se obtienen resultados similares a lo reportado por Fernández (2002b), en los profesores de primaria en lo que concierne a las subescalas de despersonalización y realización personal, mientras que en agotamiento emocional observó que las mujeres tenían mayor nivel de agotamiento emocional que los varones, hecho que no se presentó este estudio, debido probablemente a que en secundaria los niveles de exigencia no difieren en función al sexo del docente, pues entre otros factores en primaria predomina la enseñanza unidocente mientras que en secundaria es polidocente.

Respecto al contraste por tipo de colegio (Tabla 9) se observó diferencias estadísticamente significativas entre los profesores de colegios estatales y no estatales en la subescala cansancio emocional, siendo los docentes de centros educativos no estatales quienesobtuvieron un promedio de rangos más elevado, lo que significa que estos docentes se describieron como emocionalmente exhaustos por el trabajo. En la subescala despersonalización no se encontró diferencias estadísticas significativas entre los docentes de ambos tipos de colegios. Sin embargo, en la subescala realización personal, se pudo observar que existen diferencias significativas entre los docentes de colegios estatales y no estatales, siendo los docentes de centros educativos estatales quienes obtuvieron puntuaciones que indican un mayor nivel de competencia y éxito en el trabajo con los alumnos. Estos hallazgos en la subescala de realización personal son similares a los reportados por Fernández (2001) lo cual podría deberse al hecho que los colegios estatales estarían presentando un sistema de trabajo flexible que le permite al docente organizar su horario $\mathrm{O}$ exigencias laborales de manera conveniente, lo cual contrasta con las exigencias laborales de los sistemas no estatales, tales como las referidas a las exigencias de excelencia, capacitación constante o competitividad.

Cuando se realizó la comparación por tiempo de servicios (Tabla 10), no se observaron diferencias estadísticamente significativas en ninguna de las subescalas del inventario, esto indica que los niveles de cansancio emocional, despersonalización y realización personal no 
varían significativamente en función al tiempo de servicios de los profesores, tanto de colegios estatales como no estatales. Este resultado es similar a los hallazgos encontrados 'Por Fernández (2001), lo cual indicaría que los profesores tienden a desarrollar los niveles de burnout en independencia del tiempo que están laborando en el sistema educativo.

En la comparación por sexo y tipo de colegio (Tabla 11), se encontraron diferencias estadísticamente significativas entre los docentes varones y mujeres tanto de colegios estatales como de colegios no estatales en las tres subescalas. Es factible destacar que las docentes de colegios no estatales mostraron indicadores más altos en las subescalas de cansancio emocional y despersonalización, lo que estaría indicando que estas docentes se sienten emocional mente exhaustas, presentando por tanto respuestas impersonales y frías hacia sus alumnos. En la subescala de realización personal, también se encontró diferencias estadísticamente significativas, pero en este caso quienes obtuvieron los puntajes más altos fueron los docentes de colegios estatales, lo que indicaría que ellos se sienten competentes y con éxito en el trabajo con sus alumnos.

Al comparar a los profesores y las profesoras de colegios estatales (Tabla 12), se observaron diferencias significativas en las subescalas cansancio emocional y despersonalización, siendo los varones quienes obtuvieron promedios de rangos más altos, lo cual indicaría que los profesores de colegios estatales sienten mayor agotamiento emocional y presentan mayor nivel de respuestas impersonales con sus alumnos que sus colegas mujeres del mismo tipo de colegio. En cuanto a la subescala realización personal no se encontró diferencias significativas entre ambos grupos de docentes colegios estatales, posibilitando señalar que no difieren en sus sentimientos de competencia y éxito en el trabajo.

Cuando se hizo la comparación anterior, esto es en las subescalas de cansancio emocional, despersonalización, y realización personal en los docentes de colegios no estatales (Tabla 13), no se encontraron diferencias estadísticamente significativas entre los docentes varones y mujeres en ninguna de las tres subescalas, lo que indicaría que no difieren en los niveles de cansancio emocional, despersonalización y realización personal.

Al comparar a los docentes varones de colegios estatales y no estatales (Tabla 14), no se observaron diferencias estadísticas significativas en ninguna de las tres subescalas del inventario, pero al realizar la comparación entre docentes mujeres por tipo de cole-

(Tabla 15), se encontraron diferencias significativas en las subescalas cansancio emocional despersonalización y realización personal, siendo las docentes de colegios no estatales quienes mostraron niveles más altos de agotamiento emocional y despersonalización, y consecuentemente con estos hallazgos, las docentes de colegios estatales mostraron mayor nivel en sus sentimientos de competencia y éxito en su trabajo.

Cuando se comparó a los profesores de colegios estatales con las profesoras de colegios no estatales (Tabla 16), sólo se encontraron diferencias estadísticamente significativas en la subescala realización personal, observándose que los profesores de colegios estatales se sienten más realizados profesionalmente que sus colegas mujeres que laboran en colegios no estatales.

Finalmente, al comparar a las docentes de centros educativos estatales con los docentes de centros educativos no estatales (Tabla 17), solamente se observaron diferencias estadísticamente significativas en la subescala cansancio emocional, observándose que son los docentes de colegios no estatales quienes mostraron un mayor nivel de agotamiento emocional.

Con relación a las fases del burnout se encuentra que predominan en los profesores de secundaria las fases 1 y VIII, es decir los valores extremos, mientras que en los de primaria por el contrario predominan las fases VI y III, es decir los valores más cercanos a la media (Fernández, 2002a). Estos hallazgos indicarían la existencia de factores particulares 
diferenciales en los distintos niveles de enseñanza que serían los encargados de influir las condiciones para el desarrollo del burnout y que puede traducirse en hechos como el trabajar con una gran cantidad de aulas de diversos años de estudio y probablemente dada la situación económica la cantidad de carga de trabajo que poseen.

Respecto a los niveles de burnout, se observa que en el nivel alto se ubica el $32.7 \%$ de la muestra de docentes de educación secundaria, en tanto que en el estudio del nivel primario (Fernández, 2002a) se encuentra que el nivel alto corresponde al 43.2\% de los profesores de primaria, lo que estaría reflejando que las difíciles y estresantes condiciones de trabajo influyen negativamente en la salud de los profesores. En el nivel bajo de burnout se encuentra el $45.9 \%$ de los docentes de secundaria mientras que en los de primaria se encuentra el $36.3 \%$, es decir que los resultados de los indicadores de burnout están inversamente relacionados con el nivel educativo, lo cual podría estar asociado a la diferencia de recursos personales desarrollados por los profesores de cada nivel.

Sin duda que estos resultados ameritan seguir investigando lo referido al burnout en los docentes, y en general lo correspondiente al bienestar psicológico de los mismos, pues el desafío del conocimiento y desarrollo sostenible del presente milenio, encuentra en la educación uno de sus pilares fundamentales.

\section{CONCLUSIONES}

1. El Inventario «Burnout» de Maslach presenta confiabilidad por consistencia interna, por el método del Alfa de Cronbach.

2. El Inventario «Burnout» de Maslach presenta validez de constructo, tal como lo señala el análisis de factores respectivo.

3. El baremo está adaptado para ser utilizado en docentes varones y mujeres, de colegios estatales y no estatales de la ciudad de Lima, Perú.

4. Considerando el sexo no existen diferencias en los indicadores de burnout.

5. La hipótesis $\mathrm{H} 2$ es aceptada, en los profesores de colegios estatales predomina el nivel bajo y en los profesores de colegios no estatales predominan los niveles bajo y alto de burnout.

6. Considerando el tiempo de servicio no existen diferencias en las diferentes comparaciones referidas a las subescalas del inventario de «Burnout».

7. No se observaron diferencias estadísticamente significativas en ninguna de las subescalas del inventario MBI al compararse los grupos según el sexo.

8. Los docentes de centros educativos no estatales presentaron mayores niveles de cansancio emocional que sus colegas de centros educativos estatales.

9. No existen diferencias estadísticamente significativas en la subescala despersonalización entre los docentes de ambos tipos de colegios.

10. Los docentes de centros educativos estatales presentaron un mayor nivel de realización personal que sus colegas de colegios no estatales.

11. No se observaron diferencias estadísticamente significativas en ninguna de las subescalas del inventario al compararse los grupos según el tiempo de servicio.

12.Las docentes de colegios no estatales alcanzaron puntuaciones más altas en las subescalas cansancio emocional y despersonalización que sus colegas varones del mismo tipo de colegio.

13.Los profesores de centros educativos estatales presentaron mayor nivel de realización personal que sus colegas mujeres de colegios estatales. 
14. Los docentes de colegios estatales obtuvieron puntajes más elevados en las subescalas cansancio emocional y despersonalización que las docentes del mismo tipo de colegio.

15. Los profesores y las profesoras de colegios estatales no difieren significativamente en su desempeño en la subescala realización personal.

16.No existen diferencias estadísticamente significativas entre los profesores y las profesoras de colegios no estatales en ninguna de las tres subescalas del inventario.

17.Los docentes varones de colegios estatales y no estatales no difieren significativamente en el desempeño de las tres subescalas.

18.Las profesoras de colegios no estatales mostraron niveles más altos de agotamiento emocional y despersonalización que las de colegios estatales.

19. Las docentes de centros educativos estatales mostraron mayor nivel en sus sentimientos de competencia y éxito en su trabajo que sus colegas mujeres de centros educativos no estatales.

20. No se observaron diferencias significativas en el desempeño de las subescalas cansancio emocional y depersonalización entre los profesores de colegios estatales y las profesoras de colegios no estatales.

21.Los profesores de colegios estatales presentan mayor realización personal que las docentes de colegios no estatales.

22.Los docentes varones de colegios no estatales presentan mayor nivel de cansancio emocional que las docentes mujeres de colegios estatales.

23.No existen diferencias significativas en las subescalas de despersonalización y realización personal entre las profesoras de colegios estatales y los profesores de colegios no estatales.

24.Existen diferencias significativas, en las fases del burnout propuestas por Golembiewski, predominando en los profesores las fases I y VIII.

25.Existen diferencias en los niveles de burnout en los profesores de secundaria, predominando los indicadores correspondientes al nivel bajo y al nivel alto de burnout.

26.El $32.7 \%$ de los profesores de secundaria de Lima Metropolitana presentan niveles altos de burnout.

27.No existen diferencias significativas entre hombres y mujeres en las ocho fases del burnout.

28.Considerando el tipo de colegio existen diferencias significativas en las ocho fases del burnout. Predominan en los profesores de colegios estatales las fases del I al IV y en los colegios no estatales predomina la fase IV.

29. No existen diferencias significativas considerando el tiempo de servicio en las ocho fases del burnout. 


\section{REFERENCIAS BIBLIOGRÁFICAS}

Alarcón, R (1991). Métodos y diseños de investigación del comportamiento. Lima: Fondo Editorial.

Álvarez, E., y Fernández, L. (1991). El síndrome de «Burnout» o el desgaste profesional (l). Revista Asociación Española Neuropsiquiatría. XI, 39, 257-265.

American Psychological Association (2002). Manual de estilo de publicaciones. Méx.ico: Manual Moderno.

Calvete, E., Villa, A. (1999). Estrés y «burnout» docente: influencia de variables cognitivas. Revista de Educación. 319. p. 291-303.

Cherniss, C. (1980). Professional burnout in human service organization.New York: Praeger.

Fernández, M. (2001). Fuentes de presión laboral, tipo de personalidad, desgaste psíquico (burnout), satisfacción laboral y desempeño docente en profesores de educación primaria de Lima Metropolitana. Tesis para optar el Grado Académico de Doctor en Educación. UNMSM.

Fernández, M. (20o2a). Realidad psicosocial del maestro de primaria. Lima: Fondo de Desarrollo Editorial. Universidad de Lima.

Fernández, M. (2002b). Desgaste psíquico (burnout) en profesores de primaria de Lima Metropolitana. Persona. Revista de la Facultad de Psicología. Universidad de Lima. 5, 27-66

Freudenberger, H. J. (1974). Staff Bumout. Journal of Social Issues, 30,159-165.

Freudenberger, H. J. (1975). The staff burnout syndrome in alternative institutions. Psychotherapy: Theory, Research \& Practice, 12, 72-83.

Gil, P, Y Peiró, 1. (1997). Desgaste psíquico en el trabajo: El síndrome de quemarse. Madrid: Editorial Síntesis.

Golembiewski, R.T., Munzenrider, R., y Carter, D. (1983). Phases of progressive bumout and their work site covariants: Critical issues in $O D$ research and praxis. JournaJ of Applied Behavioral Science, 19, 4, 461-481.

Golembiewski, R.T., Munzenrider, R., y Stevenson, J. G. (1986). Stress in organizations: Toward a phase model of burnout. New York: Praeger.

Golembiewski, R.T., y Runtree, B. (1991).

Releasing human potential for collaboration: A social intervention targeting supervisory relationships and stress. Public Administration, 15(1), 32-45

Guerrero, E. (1999). Una investigación con docentes universitaros sobre el afrontamiento del estrés laboral y el síndrome del «quemado». OEl-Revista Iberoamericana de Educación.

Kline, P. (1986). A handbook of test construction. Introduction to psychometric designo New York: Methuen \& eo. Ltd.

Maslach, C. (1982). Understanding burnout: Definitional issues in analyzing a complex phenomenon. En W.S. Paine (Ed), Job stress and burnout. Beverly Hills, CA:Sage.

Maslach, C., y Jackson, S. (1981). The measurement of experienced burnout. Journal of Occupational Behavior. 2, 99-113.

Maslach, C., y Jackson, S. (1985). Burnout research in the social se rvices: a critique. Special issues: Burnout among social workers. Journal of social service research. 10 (1), 95- 105

Maslach, C., y Jackson, S. (1986). Maslach Burnout Inventory. California: Consulting Psychologists Press.

Maslach c., y Jackson, S (1997). Manual del Inventario «Burnout» de Maslach. Madrid: TEA Ediciones, S.A. 
Maslach c y Pines, A. (1977). The burnout syndrome in day care setting. Child careo $62,100-113$.

Muñiz, A. (1996). Psicometría. Madrid:

Editorial Pirámide.

Salgada, A., Yela, J. R., Queveda, M. P., Delgado, N., Fuentes, J. M., Sánchez, A., Sánchez, T., y Ve1asco, C. (1997). El síndrome del «Burnout»: Estudio Empírico en profesores de enseñanza primaria. Iberpsicología 2,1,1.

Topf, M., y Dillon (1989). Personality hardiness, occupational stress and burnout in critical case nurses. Research in Nursing and Health, 12, 179-186.

Vandenberghe, R. y Huberman, A.M. (1999). Understanding and preventing teacher burnout: A sourcebook of inte mational research and practice. Cambridge:

Cambridge University Press

ANEXO 1

Tabla 26. Rangos percentiles por subescalas del Inventario de "Burnout" de Malasch

\begin{tabular}{|c|c|c|c|c|}
\hline Percentil & $\begin{array}{l}\text { Cansancio } \\
\text { emocional }\end{array}$ & Depersonalización & $\begin{array}{l}\text { Realización } \\
\text { personal }\end{array}$ & Percentil \\
\hline 5 & \multirow{13}{*}{ tat } & & \multirow[t]{2}{*}{3} & 5 \\
\hline 10 & & & & 10 \\
\hline 15 & & & \multirow[t]{8}{*}{4} & 15 \\
\hline 20 & & & & 20 \\
\hline 25 & & & & 25 \\
\hline 30 & & & & 30 \\
\hline 35 & & & & 35 \\
\hline 40 & & & & 40 \\
\hline 45 & & & & 45 \\
\hline 50 & & & & 50 \\
\hline 55 & & & 5 & 55 \\
\hline 60 & & 1 & & 60 \\
\hline 65 & & & & 65 \\
\hline 70 & \multirow[t]{4}{*}{2} & & & 70 \\
\hline 75 & & & & 75 \\
\hline 80 & & & & 80 \\
\hline 85 & & 2 & & 85 \\
\hline 90 & \multirow[t]{2}{*}{3} & & & 90 \\
\hline 95 & & 3 & & 95 \\
\hline 99 & 4 & 4 & 6 & 99 \\
\hline $\mathbf{M}$ & 1.49 & 0.82 & 4.83 & $\mathbf{M}$ \\
\hline D.E & 1.02 & 0.91 & 0.91 & D.E \\
\hline
\end{tabular}

$\mathrm{N}=764$ 